\title{
Prevenção de cárie dentária por bochechos com flúor em município com água fluoretada
}

\author{
Maria Luiza Hiromi Iwakura e Maria Celeste Morita ${ }^{1}$
}

Como citar Iwakura MLH, Morita MC. Prevenção de cárie dentária por bochechos com flúor em município com água fluoretada. Rev Panam Salud Publica. 2004;15(4):256-61.

RESUMO Objetivo. Comparar a prevalência de cárie dentária em escolares que participaram de um programa semanal de bochecho com fluoreto de sódio a 0,2\% com a prevalência de cárie em escolares que não participaram do programa.

Métodos. Estudo transversal na Cidade de Londrina, Estado do Paraná, Brasil, cuja população consome água fluoretada. Foram examinados 367 escolares de 12 anos: 190 participantes $(51,8 \%)$ e 177 não participantes $(48,2 \%)$ do programa semanal de bochecho. Os indices utilizados foram o de dentes cariados, perdidos e obturados e o de superfícies cariadas, perdidas e obturadas. Os exames foram conduzidos por três examinadores e a concordância no diagnóstico de cárie foi quase perfeita $(K=0,90)$. A cárie dentária foi considerada variável dependente. As variáveis independentes foram: a participação ou não no programa, estudar em escola pública ou privada, escovação dentária, quantidade de dentifrício utilizada, ingestão de doces e consulta ao dentista.

Resultados. O indice de dentes cariados, perdidos e obturados aos 12 anos foi 0,85 $\pm 0,059$ $(0,70 \pm 0,060$ para não participantes e 1,0 $\pm 0,058$ para participantes $)$. $O$ indice de superfícies cariadas, perdidas e obturadas foi $1,16 \pm 0,017$, variando de 0,34 a 1,66. Na análise bivariada, estiveram estatisticamente associados $(\mathrm{P}<0,05)$ com a presença de cárie: estudar em escola pública, participar no programa de bochecho e ingerir doces entre as refeições mais de uma vez por dia. Na análise de regressão logística multivariada, mantiveram-se associados com a presença de cárie estudar em escola pública $(\mathrm{P}=0,0004)$ e ingerir doces $(\mathrm{P}=0,0010)$.

Conclusões. O programa de bochecho com flúor não esteve associado a menor prevalência de cárie, seja em escolas públicas ou privadas. Entretanto, outros estudos são necessários para análise de custo-efetividade do programa em populações com maior prevalência da doença. No nível de prevalência de cárie observado, o recurso destinado ao programa de bochecho com flúor poderia financiar outras ações de promoção de saúde.

Palavras-chave Cárie dentária, escola pública, flúor.

Universidade Estadual de Londrina, Departamento de Medicina Oral e Odontologia Infantil. Correspondência e pedidos de separatas devem ser enviados a Maria Celeste Morita no seguinte endereço: Rua Pernambuco 540, CEP 86020-070, Londrina, PR, Brasil. E-mail: oscar@sercomtel.com. br (Maria Luiza Hiromi Iwakura) e moritamc@ sercomtel.com.br (Maria Celeste Morita).
Diversos estudos têm tentado explicar o declínio na prevalência da cárie dentária em crianças de países desenvolvidos, especialmente a partir da década de 1970. Naquela década, alguns países começaram a reavaliar o papel dos programas de bochecho com flúor, método muitas vezes associado a outras formas de aplicação tópica de flúor, no combate às cáries (1-4).

Inicialmente, a estratégia de combate às cáries com programas comuni- 
tários de bochecho com flúor foi altamente eficiente. Um estudo sobre programa de bochecho com fluoreto de sódio a 0,2\% em escolares de Joanesburgo, de região com água não fluoretada, mostrou redução de aproximadamente $15 \%$ na prevalência de cárie dentária (5). Yamaguchi et al. (6), no Japão, em região onde a água de abastecimento não era fluoretada, concluíram que os escolares de 12 anos que interromperam após 6 anos um programa de bochecho com flúor tiveram uma elevação no índice de dentes cariados, perdidos e obturados (CPOD) em relação àqueles que continuaram no programa.

Atualmente, contudo, em sociedades nas quais a maior parte da população utiliza dentifrícios com flúor, e onde os níveis de cárie dentária são baixos, os programas comunitários mostram pouca eficiência (7). Birkeland et al. (8), em estudo sobre possíveis fatores que poderiam estar associados ao declínio de cárie dentária, relatam que os programas de bochecho com flúor em escolares podem ter influenciado o declínio da prevalência de cárie em escolares de 8 a 11 anos na década de 1960. Já a partir da década de 1970, o declínio na prevalência de cárie pode estar relacionado à livre comercialização de dentifrícios e outros compostos contendo flúor e a um melhor nível de escolaridade dos pais. Assim, o custo-benefício dos programas de bochecho tem sido questionado, especialmente em áreas de baixa prevalência de cárie (1, 9-12). Em geral, os bochechos com flúor são efetivos em áreas com concentrações insuficientes de flúor na água, e para as crianças que recebem terapias de flúor inadequadas.

No Brasil, a aplicação de bochechos de fluoreto de sódio a $0,2 \%$ tem sido um dos métodos mais empregados para a prevenção da cárie depois da fluoretação da água de abastecimento público. Em Londrina, o programa de bochecho semanal com flúor, que completou 20 anos em 2001, abrange 248872 escolares de 6 a 12 anos. Durante esse período, vários levantamentos epidemiológicos de cárie dentária foram realizados no Município, porém sem a finalidade específica de avaliar o impacto do programa de bochecho semanal com flúor. Sabe-se que, de 1981 a 2001, houve uma redução de $84 \%$ no CPOD em escolares do Município (13, 14). Na zona urbana de Londrina, a prevalência de cárie em crianças de 12 anos é, atualmente, de 1,11 CPOD.

Dessa forma, o objetivo do presente estudo foi comparar a prevalência de cárie dentária em escolares da Cidade de Londrina que participaram do programa semanal de bochecho com fluoreto de sódio a $0,2 \%$ com a prevalência de cárie em escolares que não participaram do programa.

\section{MATERIAIS E MÉTODOS}

A Cidade de Londrina, localizada no norte do Estado do Paraná, tem uma população de 447065 habitantes (15). A fluoretação da água de abastecimento público no Município teve início em 1972, e toda a população urbana consome água fluoretada. A concentração média dos níveis de flúor é de 0,77 ppm.

Para avaliar a prevalência de cárie em escolares que participavam ou não do programa municipal de bochechos com fluoreto de sódio a $0,2 \%$, foi desenvolvido um estudo transversal controlado de conveniência. A população do estudo incluiu crianças de 12 anos de quatro escolas (duas públicas e duas privadas): uma escola pública e duas privadas foram selecionadas a partir do critério de proximidade geográfica. A única escola pública que não participava do programa foi incluída para possibilitar a comparação entre os grupos. Nas escolas onde o programa era desenvolvido, foram incluídas todas as crianças que participaram do programa de bochechos com fluoreto de sódio a 0,2\% dos 6 aos 12 anos; na escola que não participava do programa, foram selecionadas todas as crianças que não haviam sido expostas ao programa de bochechos dos 6 aos 12 anos.

A estimativa prévia do total de escolares a serem examinados era de 458 . No primeiro semestre de 2001, foram examinadas 367 crianças $(80,1 \%)$. Das $91(19,9 \%)$ crianças não examinadas,
69 usavam aparelho ortodôntico fixo, 14 não tiveram autorização dos pais para o exame e oito faltaram nos dias de exame. Quando o aluno faltava no dia marcado, o examinador retornava mais duas vezes, na tentativa de conseguir realizar o exame.

A população de estudo foi constituída de 367 escolares de 12 anos de idade (196 meninos e 171 meninas) matriculados nas escolas da rede pública e privada de ensino, localizadas na zona urbana do Município. A idade de 12 anos foi escolhida por ser passível de comparações com estudos de prevalência de cárie dentária anteriormente desenvolvidos no Município e por ser a idade de referência recomendada pela Organização Mundial de Saúde (OMS) para comparações com outras regiões do país e do mundo (16).

Para a determinação da prevalência de cárie dentária nesta população foram utilizados o CPOD e o índice de superfícies cariadas, perdidas e obturadas (CPOS). Os critérios diagnósticos adotados para o CPOD e o CPOS foram os descritos no Manual de Levantamentos Básicos em Saúde Bucal (16), adaptando-se o uso da sonda exploradora romba em substituição à sonda CPI, recomendada para levantamentos epidemiológicos desta natureza.

O projeto foi aprovado pela Comissão de Ética em Pesquisa da Universidade Estadual de Londrina. Também foi enviada uma carta explicativa do projeto para cada pai ou responsável pelo aluno a ser examinado, juntamente com duas vias do termo de consentimento livre e esclarecido.

Previamente aos exames clínicos, os cirurgiões dentistas examinadores e anotadores participaram de um treinamento para padronizar e nivelar a coerência do diagnóstico de cárie segundo os critérios da OMS (16). Os exames foram realizados por três examinadores. Cada examinador realizou o exame em 10 escolares, observandose as superfícies dentárias, num total de 1175 superfícies examinadas. Para avaliação da concordância entre os examinadores, utilizou-se o coeficiente Kappa de Cohen. O índice de concordância obtido entre os examinadores para presença e ausência de cárie 
dentária foi de $\mathrm{K}=0,90$, valor considerado como concordância quase perfeita.

Os exames clínicos foram realizados nas escolas com sonda exploradora romba, espelho plano e espátula de madeira, sob luz natural. As superfícies dentárias foram secas com gaze para melhorar as condições diagnósticas.

Elaborou-se um questionário com perguntas fechadas para levantar informações adicionais quanto a condições socioeconômicas e exposição prévia a outras fontes de flúor, a fim de se controlar fatores de confusão que poderiam influenciar e interferir nos resultados. Para se estabelecer a quantidade de dentifrício utilizado, optouse pela escala de Levy e Zarei-M (17). Um pré-teste do questionário foi realizado com 20 alunos. Depois do préteste foram feitos os ajustes necessários para melhorar a compreensão do questionário por parte dos alunos e do entrevistador.

Considerou-se como variável dependente a presença de cárie dentária. As variáveis independentes foram: estudar em escola pública ou privada, participar ou não no programa semanal de bochecho com flúor, a freqüência da escovação, a quantidade de dentifrício utilizada, a freqüência de ingestão de doces entre as refeições e a data da última consulta ao dentista.

Para a elaboração dos bancos de dados utilizou-se o programa Epi Info versão 6.04d (18). Para a análise de regressão logística multivariada utilizou-se o Statistical Analysis System (SAS) (19).

\section{RESULTADOS}

A tabela 1 mostra a distribuição das variáveis quanto à participação no programa de bochecho com flúor. Observou-se resultado estatisticamente significativo (ao nível de 5\%) em relação à presença de cárie dentária para participantes do programa de bochecho com flúor $\left(P=0,0059, \chi^{2}\right)$ na análise bivariada. Porém, na análise multivariada, essa associação não foi significativa ( $P=0,0154$, teste de Wald $)$.

A divisão em escolas públicas e privadas teve como objetivo comparar

TABELA 1. Distribuição das variáveis quanto a participação ou não no programa semanal de bochecho com fluoreto de sódio a 0,2\%, Londrina (PR), Brasil, 2001

\begin{tabular}{|c|c|c|c|c|c|}
\hline \multirow[b]{3}{*}{ Variáveis } & \multirow{3}{*}{$\begin{array}{l}\text { Crianças } \\
\text { examinadas }\end{array}$} & \multicolumn{4}{|c|}{$\begin{array}{c}\text { Participação no programa } \\
\text { de bochecho }\end{array}$} \\
\hline & & \multicolumn{2}{|c|}{ Não } & \multicolumn{2}{|c|}{ Sim } \\
\hline & & No. & $\%$ & No. & $\%$ \\
\hline \multicolumn{6}{|l|}{ Tipo de escola } \\
\hline Pública & 183 & 81 & 22,0 & 102 & 27,8 \\
\hline \multirow{2}{*}{ Privada } & 184 & 96 & 26,2 & 88 & 24,0 \\
\hline & 367 & 177 & 48,2 & 190 & 51,8 \\
\hline \multicolumn{6}{|l|}{ Cárie dentária } \\
\hline Presente & 133 & 51 & 13,9 & 82 & 22,4 \\
\hline \multirow[t]{2}{*}{ Ausente } & 234 & 126 & 34,3 & 108 & 29,4 \\
\hline & 367 & 177 & 48,2 & 190 & 51,8 \\
\hline \multicolumn{6}{|l|}{ Hábito de escovar os dentes } \\
\hline Às vezes & 5 & 2 & 0,6 & 3 & 0,8 \\
\hline \multirow[t]{2}{*}{ Sim } & 362 & 175 & 47,7 & 187 & 50,9 \\
\hline & 367 & 177 & 48,3 & 190 & 51,7 \\
\hline \multicolumn{6}{|l|}{ Freqüência da escovação } \\
\hline$\leq 1$ vez ao dia & 35 & 17 & 4,6 & 18 & 4,9 \\
\hline \multirow[t]{2}{*}{$\geq 2$ vezes ao dia } & 332 & 160 & 43,6 & 172 & 46,9 \\
\hline & 367 & 177 & 48,2 & 190 & 51,8 \\
\hline \multicolumn{6}{|l|}{ Quantidade de dentifrício } \\
\hline Não recomendada & 314 & 149 & 40,6 & 165 & 45,0 \\
\hline \multirow[t]{2}{*}{ Recomendada } & 53 & 28 & 7,6 & 25 & 6,8 \\
\hline & 367 & 177 & 48,2 & 190 & 51,8 \\
\hline \multicolumn{6}{|l|}{ Ingestão de doce entre as refeições } \\
\hline Não & 18 & 5 & 1,4 & 13 & 3,6 \\
\hline \multirow[t]{2}{*}{ Sim } & 349 & 172 & 46,8 & 177 & 48,2 \\
\hline & 367 & 177 & 48,2 & 190 & 51,8 \\
\hline \multicolumn{6}{|l|}{ Freqüência de ingestão de doce } \\
\hline Alta (mais do que uma vez ao dia) & 55 & 23 & 6,3 & 32 & 8,8 \\
\hline \multirow[t]{2}{*}{ Baixa ou regular } & 312 & 154 & 41,9 & 158 & 43,0 \\
\hline & 367 & 177 & 48,2 & 190 & 51,8 \\
\hline \multicolumn{6}{|l|}{ Última consulta ao dentista } \\
\hline Nunca/acesso irregular & 93 & 33 & 9,0 & 60 & 16,3 \\
\hline \multirow{2}{*}{ Acesso regular ( $<1$ ano $)$} & 274 & 144 & 39,2 & 130 & 35,5 \\
\hline & 367 & 177 & 48,2 & 190 & 51,8 \\
\hline \multicolumn{6}{|l|}{ Motivo da consulta ${ }^{a}$} \\
\hline Urgência/tratamento & 123 & 39 & 10,6 & 84 & 22,8 \\
\hline Controle/manutenção & 232 & 136 & 37,0 & 96 & 26,1 \\
\hline \multirow[t]{2}{*}{ Outros } & 5 & 1 & 0,2 & 4 & 1,0 \\
\hline & 360 & 176 & 47,8 & 184 & 49,9 \\
\hline \multicolumn{6}{|l|}{ Tipo de serviçob } \\
\hline Público & 140 & 61 & 16,6 & 79 & 21,5 \\
\hline Privado/convênios & 217 & 114 & 31,0 & 103 & 28,0 \\
\hline & 357 & 175 & 47,6 & 182 & 49,5 \\
\hline
\end{tabular}

grupos de condições socioeconômicas diferentes. A renda média familiar dos estudantes das escolas privadas era três vezes maior do que a dos estudantes da rede pública. Portanto, essa diferença foi considerada na análise dos dados. Na escola pública, 87 crianças $(23,7 \%)$ apresentaram cárie, contra 46 crianças $(12,5 \%)$ na escola privada. A associação entre a presença de cárie dentária e estudar em escola pública foi significativa ao nível de 5\% ( $P=$ 0,0001 ).

Analisando-se o CPOD por escola, observou-se um valor mínimo de $0,26 \pm 0,050$ em uma das escolas privadas; o valor máximo observado foi de $1,22 \pm 0,066$ em uma das escolas públicas. $\mathrm{O}$ valor médio do CPOD para a população analisada foi de $0,85 \pm 0,059$ 
TABELA 2. Valores médios dos índices de dentes cariados, perdidos e obturados e superfícies cariadas, perdidas e obturadas em escolas públicas e privadas, Londrina (PR), Brasil, 2001

\begin{tabular}{lccccc}
\hline & \multicolumn{2}{c}{$\begin{array}{c}\text { Dentes cariados, } \\
\text { perdidos e obturados }\end{array}$} & & \multicolumn{2}{c}{$\begin{array}{c}\text { Superfícies cariadas } \\
\text { perdidas e obturadas }\end{array}$} \\
\cline { 2 - 3 } & Média & $\begin{array}{c}\text { Desvio } \\
\text { padrão }\end{array}$ & & Média & $\begin{array}{c}\text { Desvio } \\
\text { padrão }\end{array}$ \\
\hline Escola pública & 1,18 & 0,063 & & 1,61 & 0,021 \\
Escola privada & 0,52 & 0,053 & & 0,68 & 0,013 \\
Geral & 0,85 & 0,059 & & 1,16 & 0,017 \\
\hline
\end{tabular}

(tabela 2), bem abaixo da meta estabelecida pela OMS para o ano 2000, que era de no máximo 3 para crianças de 12 anos (20). A tabela 3 mostra a distribuição das crianças de escolas públicas e privadas e das crianças que participaram ou não do programa semanal de bochechos em termos do CPOD.

Em relação ao CPOS, encontramos um valor mínimo de 0,34 $\pm 0,009$ para uma escola privada e máximo de 1,66 \pm 0,020 para uma escola pública. É importante ressaltar que somente os alunos de escolas públicas apresentaram superfícies perdidas (extraídas ou com extração indicada), denotando, mais uma vez, deficiências na atenção odontológica dessa população. O grupo dos participantes do programa de bochecho com flúor apresentou percentual de $74,0 \%$ de superfícies obturadas, contra $71,6 \%$ dos não participantes. As superfícies perdidas representaram
1,0\% para participantes e 3,4\% para não participantes. Quanto ao componente superfícies cariadas, em ambos os grupos $25,0 \%$ das crianças apresentavam a doença.

Praticamente todas as crianças $(98,6 \%)$ declararam escovar os dentes. Em relação à freqüência da escovação, $35(9,6 \%)$ relataram escovar uma vez ao dia ou às vezes, e $332(90,4 \%)$ disseram que escovavam duas vezes ou mais ao dia. Para escovar os dentes, $359(97,8 \%)$ dos entrevistados informaram utilizar a escova e o dentifrício. Quase todos os dentifrícios comercializados no Brasil possuem flúor. Do total, 314 dos alunos $(85,6 \%)$ utilizavam quantidades de dentifrício acima do recomendado de acordo com a escala de Levy e Zarei-M (17); 53 (14,4\%) utilizavam quantidades corretas.

A maioria das crianças indicou o hábito de consumir doces entre as
TABELA 3. Distribuição dos escolares de 12 anos em relação ao índice de dentes cariados, perdidos e obturados, Londrina (PR), Brasil, 2001

\begin{tabular}{lccr}
\hline & \multicolumn{3}{c}{ Índice de dentes cariados, } \\
perdidos e obturados (\%) \\
\cline { 2 - 4 } \multicolumn{1}{c}{ Variável } & 0 & $1-3$ & $>3$ \\
\hline Escola & & & \\
$\quad$ Pública & 52,5 & 36,0 & 11,5 \\
$\quad$ Privada & 75,0 & 21,2 & 3,8 \\
Participante do programa & & & \\
de bochecho com flúor & & & \\
$\quad$ Sim & 58,6 & 34,8 & 8,4 \\
Não & 71,2 & 22,0 & 6,8 \\
\hline
\end{tabular}

refeições $(95,0 \%)$. A periodicidade de consumo foi de uma vez por semana $(12,9 \%)$, duas vezes por semana $(18,0 \%)$, três a quatro vezes por semana $(25,5 \%)$, uma vez ao dia $(22,0 \%)$, duas a três vezes ao dia $(10,6 \%)$, mais do que três vezes ao dia $(5,1 \%)$ ou só nos finais de semana $(6,5 \%)$. Consumir alimentos cariogênicos entre as refeições mais de uma vez ao dia apresentou associação estatisticamente significativa com a presença de cárie dentária $(P=0,0013)$.

$\mathrm{Na}$ análise bivariada, apresentaram associação estatisticamente significativa com a presença de cárie dentária: estudar em escola pública $(P<0,0001)$; participar do programa de bochecho com flúor ( $P=0,0059)$; e freqüência de ingestão de doces entre as refeições $(P=0,0013)$. Na análise multivariada, mantiveram-se associados com a presença de cárie dentária o tipo da escola $(P=0,0004)$ e a freqüência de ingestão de doces entre as refeições $(P=0,0010)$. Além disso, a significância do efeito da interação entre a participação no programa de bochecho e o tipo de escola $(P=0,0154)$ e a não significância da participação no programa de bochecho $(P=0,1277)$ sugerem que a presença de cárie dentária está associada com estudar na escola pública ou privada, e não com o programa de bochecho.

\section{DISCUSSÃO}

No presente estudo, consumir doces entre as refeições e estudar em escola pública foram os principais determinantes da presença de cárie. Diversos autores apontam para o consumo de açúcares como uma das principais causas do aparecimento de cárie dentária $(21,22)$. Em relação a freqüentar a escola pública, as crianças nesse tipo de escola freqüentavam menos o dentista e utilizavam mais os serviços municipais de odontologia. O Município de Londrina apresenta uma boa estrutura de clínicas odontológicas do setor público, que oferece atendimento odontológico gratuito à população de 0 a 14 anos. Entretanto, os casos de cárie dentária com comprometimento endodôntico não são tratados na rede básica de serviços, fator que pode ter 
influenciado a existência de superfícies perdidas ou com extração indicada em alunos de escolas públicas.

O programa de bochecho com flúor não esteve associado a menor prevalência de cárie, tanto em escolas públicas como privadas. O trabalho do programa é realizado por técnicos em higiene dental e auxiliares de odontologia que saem das unidades básicas de saúde, em média, duas vezes por semana para realizar o bochecho com fluoreto de sódio a $0,2 \%$ nas escolas. Para calcular o custo mensal do programa de bochecho - $\mathrm{R} \$ 1,14$ por criança - foram considerados a média dos vencimentos por dias trabalhados no programa das auxiliares de odontologia e técnicas em higiene dental, o custo de produção do fluoreto de sódio a $0,2 \%$ e o custo de transporte. Considerando-se um total de três bochechos por mês, o custo em 1 ano seria de $\mathrm{R} \$ 284$ 903,83. Comparativamente, um programa de educação para a saúde e promoção do auto-cuidado, com distribuição de um tubo de dentifrício com flúor e uma escova de dentes para cada criança, no mesmo período, custaria $\mathrm{R} \$ 0,46$. O valor assume importância maior quando comparado com procedimentos de longo alcance social, como é o caso de vacinas, que custam $R \$ 0,50$ por dose.

Considerando que os recursos destinados à saúde pública devem ser aplicados com extrema parcimônia, o investimento no programa de bochechos no Município de Londrina poderia ser direcionado para outras ações de promoção de saúde. Os presentes resultados sugerem que a iniciativa não contribuiu para a melhoria da saúde bucal da população estudada, e que outros fatores, como a presença de flúor na água, ou o acesso a dentifrícios com flúor, podem estar implicados na baixa prevalência de cárie dentária observada. Entretanto, outros estudos são necessários para análise de custo-efetividade do programa em populações com maior prevalência da doença. No nível observado de prevalência de cárie, o recurso destinado ao programa de bochecho com flúor poderia financiar outras ações de promoção da saúde.

\section{REFERÊNCIAS}

1. Ripa LW. A critique of topical fluoride methods (dentifrices, mouthrinses, operator-, and self-applied gels) in an era of decreased caries and increased fluorosis prevalence. J Public Health Dent. 1991;51(1):23-41.

2. Ripa LW. Rinses for the control of dental caries. Int Dent J. 1992;42(suppl 1):263-9.

3. Bratthall D, Petersson GH, Sundberg H. Reasons for the caries decline: what do the experts believe? Eur J Oral Sci. 1996;104(4 Pt 2): 416-22.

4. Künzel W, Fischer T, Lorenz R, Brühmann S. Decline of caries prevalence after the cessation of water fluoridation in the former East Germany. Community Dent Oral Epidemiol. 2000;28(5):382-9.

5. Chikte UM, Lewis HA, Rudolph MJ. The effectiveness of a school-based fluoride mouthrinse programme. J Dent Assoc S Afr. 1996; 51(11):697-700.

6. Yamaguchi N, Saito T, Oho T, Sumi Y, Yamashita $Y$, Koga T. Influence of the discontinuation of a school-based, supervised fluoride mouthrinsing programme on the prevalence of dental caries. Community Dent Health. 1997;14(4):258-61.

7. Forss H. Efficiency of fluoride programs in the light of reduced caries levels in young populations. Acta Odontol Scand. 1999;57(6):34851.

8. Birkeland JM, Haugejorden O, Von Der Fehr FR. Some factors associated with the caries decline among Norwegian children and ado- lescents: age-specific and cohort analyses. Caries Res. 2000; 34(2):109-16.

9. Petersson GH. Fluoride mouthrinses and fluoride varnishes. Caries Res. 1993;27(suppl 1): 35-42.

10. Wang N. Government policies on fluoride utilization in the Nordic countries. Acta Odontol Scand. 1999;57(6):342-7.

11. World Health Organization. Fluorides and oral health. Geneva: WHO; 1994. (WHO Technical Report Series).

12. Organización Panamericana de la Salud. Recomendaciones para la vigilancia y monitoreo de fluoruros en la prevención de la caries dental. Washington, D.C.: OPS; 1999. [Documento HSP/HSO-028-99 (CF)].

13. Morita MC, Gonini CJ, Iwakura ML, Barczynsc EMF. Cárie dentária: declínio de prevalência em crianças de 6 a 12 anos em Londrina. Inf Epidemiol SUS. 1996;4:55-60.

14. Secretaria Municipal de Saúde, Diretoria de Ações em Saúde, Gerência de Odontologia. Levantamento epidemiológico de cárie nas idades de 3, 5 e 12 anos e fluorose dentária na idade de 12 anos, em escolares da rede pública e particular de ensino, zona urbana e rural, em Londrina, Paraná [relatório técnico]. Londrina: Secretaria Municipal da Saúde; 2001.

15. Instituto Brasileiro de Geografia e Estatística. Censo 2000 - resultados preliminares. Disponível em: http://www.ibge.gov.br/ibge/ estatistica/populacao/censo2000/universo. php. Acessado em 20 de janeiro de 2002.

16. Organização Mundial da Saúde. Levantamentos básicos em saúde bucal. $4^{a}$ Ed. São Paulo; Livraria Editora Santos; 1999.

17. Levy SM, Zarei-M Z. Evaluation of fluoride exposures in children. J Dent Child. 1991; 58(6):467-73.

18. Dean AG, Dean JA, Burton AH, Dicker RC. Epi Info Version 6: a word processing, database, and statistics system for epidemiology on microcomputers. Atlanta: $\mathrm{CDC} / \mathrm{WHO}$; 1998.

19. SAS Institute Inc. SAS procedures guide, version 6. 4th ed. Volumes 1 and 2. Cary, North Carolina: SAS Institute Inc; 1989.

20. Global goals for oral health by the year 2000 . Federation Dentaire Internationale. Int Dent J 1982;32(1):74-7.

21. Peres KGA, Bastos JRM, Latorre MRDO. Severidade de cárie em crianças e relação com aspectos sociais e comportamentais. Rev Saude Publica. 2000;34(4):402-8.

22. Tomita NE, Nadanovsky P, Vieira ALF, Lopes ES. Preferências por alimentos doces e cárie dentária em pré-escolares. Rev Saude Publica. 1999;33(6):542-6.

Manuscrito recebido em 20 de junho de 2003. Aceito em versão revisada em 30 de dezembro de 2003. 
ABSTRACT Objective. To compare the prevalence of dental caries in two groups of schoolchildren: (1) schoolchildren participating in a weekly $0.2 \%$ sodium fluoride mouth-rinsing program and (2) schoolchildren not participating in the program.

\section{Fluoride mouth-rinsing to prevent dental caries in a Brazilian municipality with fluoridated drinking water}

Methods. This cross-sectional study was conducted in the city (municipality) of Londrina, in the state of Paraná, Brazil; the city has fluoridated drinking water. We examined 367 12-year-old children: 190 participants in the weekly mouth-rinsing program $(51.8 \%)$ and 177 nonparticipants $(48.2 \%)$. The prevalence of caries was determined based on the scores for decayed, missing, and filled teeth and for decayed, missing, and filled surfaces. The examinations were performed by three examiners, with nearly perfect agreement in their diagnosis of caries (kappa $=0.90)$. The dependent variable was dental caries. The independent variables were: participation or nonparticipation in the mouth-rinsing program, attending a private school or a public school, frequency of tooth-brushing, amount of toothpaste used, consumption of sweets between meals, and visits to the dentist.

Results. The decayed, missing, and filled teeth score (mean and standard deviation) at 12 years of age was $0.85 \pm 0.059$ overall, $1.0 \pm 0.058$ for program participants, and $0.70 \pm 0.060$ for nonparticipants. The decayed, missing, and filled surfaces score (mean and standard deviation) was $1.16 \pm 0.017$, with it ranging from a low of 0.34 in one private school to a high of 1.66 in one public school. Bivariate analysis showed an association between caries $(P<0.05)$ and the following variables: attending a public school, participating in the mouth-rinsing program, and consuming sweets between meals more than once a day. In the multivariate analysis, attending a public school $(P=0.0004)$ and consuming sweets $(P=0.001)$ remained associated with the presence of caries.

Conclusions. The weekly mouth-rinsing program was not associated with a decreased prevalence of caries, in either the public schools or the private schools. However, additional research is needed to assess the cost-effectiveness of fluoride mouthrinsing programs in populations with a higher prevalence of caries. Given the caries prevalence that we found in the schoolchildren whom we studied, the resources allocated to the fluoride mouth-rinsing program for them should probably be used to pay for other health-promotion activities with them.

\section{Clasificación Internacional de Enfermedades Aplicada a Odontología y Estomatología (CIE-OE), 3. ${ }^{a}$ ed.}

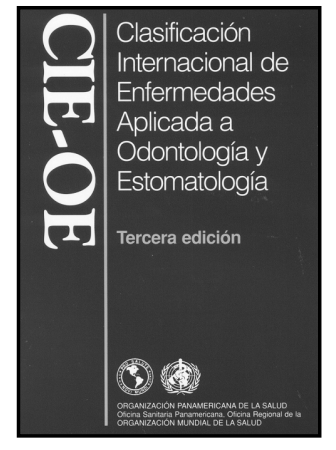

1996, 238 pp., ISBN 9275315620 Código: PC 562, Precio: US\$ 32.00/ US\$ 22.00 en América Latina y el Caribe
Esta tercera edición de la Clasificación Internacional de Enfermedades Aplicada a Odontología y Estomatología (CIE-OE) tiene por objeto proveer bases prácticas y convenientes para codificar y clasificar datos relativos a los trastornos bucales y dentarios. La CIE-OE se deriva directamente de la Décima Revisión de la Clasificación Internacional de Enfermedades (CIE-10), y ha sido concebida como parte de la "familia"de clasificaciones de enfermedades y problemas relacionados con la salud.

En la CIE-OE se incluyen todas las enfermedades y trastornos que se presentan en la cavidad bucal y estructuras adyacentes, tienen manifestaciones en ellas o están asociadas con las mismas. La mayoría de las clasificaciones provistas por la CIE-10 han sido subdivididas y expandidas para incluir un quinto carácter. El propósito de la inclusión de este quinto carácter es llamar la atención de los profesionales de la salud bucal sobre la necesidad de realizar un diagnóstico detallado para cada paciente a fin de proveer un registro estandarizado para todos los diagnósticos y facilitar la comparación de los datos a nivel internacional. Se espera que la CIE-OE contribuya en forma importante a la recolección de datos epidemiológicos sobre las enfemedades bucales menos frecuentes, para las cuales los métodos de vigilancia son impracticables. 